Physics

Physics Research Publications

Purdue University

Year 2005

\title{
Ground- and excited-state electronic structure of an iron-containing molecular spin photoswitch
}

J. H. Rodriguez

This paper is posted at Purdue e-Pubs.

http://docs.lib.purdue.edu/physics_articles/219 


\title{
Ground- and excited-state electronic structure of an iron-containing molecular spin photoswitch
}

\author{
Jorge H. Rodriguez ${ }^{a)}$ \\ Department of Physics, Purdue University, West Lafayette, Indiana 47907-2036
}

(Received 15 June 2005; accepted 14 July 2005; published online 8 September 2005)

\begin{abstract}
The electronic structure of the cation of $\left[\mathrm{Fe}(\mathrm{ptz})_{6}\right]\left(\mathrm{BF}_{4}\right)_{2}$, a prototype of a class of complexes that display light-induced excited-state spin trapping (LIESST), has been investigated by time-independent and time-dependent density-functional theories. The density of states of the singlet ground state reveals that the highest occupied orbitals are metal centered and give rise to a low spin configuration $\mathrm{Fe}^{2+}\left(3 d_{x y}^{\uparrow \downarrow} 3 d_{x z}^{\uparrow \downarrow} 3 d_{y z}^{\uparrow \downarrow}\right)$ in agreement with experiment. Upon excitation with light in the $2.3-3.3 \mathrm{eV}$ range, metal-centered spin-allowed but parity-forbidden ligand field (LF) antibonding states are populated which, in conjunction with electron-phonon coupling, explain the experimental absorption intensities. The computed excitation energies are in excellent agreement with experiment. Contrary to simpler models we show that the LF absorption bands, which are important for LIESST, do not originate in transitions from the ground to a single excited state but from transitions to manifolds of nearly degenerate excited singlets. Consistent with crystallography, population of the LF states promotes a drastic dilation of the ligand cage surrounding the iron. (C) 2005 American Institute of Physics. [DOI: 10.1063/1.2018631]
\end{abstract}

\section{INTRODUCTION}

Some iron-containing complexes have been found to exhibit optically and thermally induced spin crossovers whereby a transition is made from their singlet ground state to a long-lived (order of days) metastable high spin state. ${ }^{1,2}$ In particular, upon excitation with green light, crystalline (Fig. 1) and powder forms of $\left[\mathrm{Fe}(\mathrm{ptz})_{6}\right]\left(\mathrm{BF}_{4}\right)_{2}$ make spinforbidden transitions from the low spin (LS) $S=0$ state to a high spin (HS) $S=2$ metastable state which becomes trapped below a critical temperature of about $50 \mathrm{~K}$. This phenomenon is known as light-induced excited-state spin trapping (LIESST). ${ }^{1}$ Such spin transitions are also observed upon thermal excitation at about $135 \mathrm{~K}$ and are accompanied by dramatic structural rearrangements whereby a rombohedral $\rightarrow$ triclinic phase transition also occurs. ${ }^{3,4}$ Concomitant with the magnetic transitions are significant rearrangements of orbital occupation and spin configuration of the central $\mathrm{Fe}^{2+}$ valence shell which, in a cubic ligand field, can be described as $\quad d_{x y}^{\uparrow \downarrow} d_{x z}^{\uparrow \downarrow} d_{y z}^{\uparrow \downarrow} d_{x^{2}-y^{2}} d_{z^{2}} \rightarrow d_{x y}^{\uparrow \downarrow} d_{x z}^{\uparrow} d_{y z}^{\uparrow} d_{x^{2}-y^{2}}^{\uparrow} d_{z^{2}}^{\uparrow}$. Similarly, the structural transitions are associated with unusually large $\mathrm{Fe}-\mathrm{N}$ bond elongations of about $0.2 \AA \AA^{3,4}$

Due to the ability to control spin transitions and color changes in $\left[\mathrm{Fe}(\mathrm{ptz})_{6}\right]\left(\mathrm{BF}_{4}\right)_{2}$ by means of optical excitation, this and similar complexes are seen as photoswitches for potential use in novel molecular-level memory storage or liquid-crystal display applications. ${ }^{2,5}$ Some progress has been made in understanding the basic mechanism of LIESST, mostly based on optical and other spectroscopies. ${ }^{1,2,6}$ The qualitative mechanism for high spin trapping in $\left[\mathrm{Fe}(\mathrm{ptz})_{6}\right]$ $\times\left(\mathrm{BF}_{4}\right)_{2}$ has been described as an excitation from the singlet ground state to other short-lived, higher-energy singlets

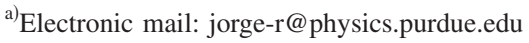

(Fig. 2). This is followed by spin-forbidden intersystem crossing to an intermediate lower-energy triplet which, mediated by spin-orbit coupling, changes the total spin of the system by one unit. Another spin-forbidden intersystem crossing brings the system to the metastable quintet state where it is subsequently trapped below its critical temperature. ${ }^{1,2,6}$

Most of the optical experiments have focused on excitations of the initial $S=0$ and final $S=2$ states of LIESST complexes because these states can be trapped by controlling the temperature and their crystallographic structures have also been determined. ${ }^{2-4,6}$ However, further theoretical understanding is needed about the detailed electronic structure of LIESST-exhibiting complexes. In particular; one wishes to elucidate their ground- and excited-state electronic structures, the origin of the optical absorption associated with the vertical singlet excited states, and how the excited-state electronic structures are related to the dramatic geometric changes observed by crystallography. ${ }^{3,4}$ Such understanding will likely lead to the design and synthesis of new LIESST materials with higher critical temperatures (below which the high spin state is effectively trapped) for use in potentially novel memory storage or display technologies. ${ }^{1,2,6}$ In this work, after a brief description of the ground-state electronic structure, we focus on the computation of singlet $\rightarrow$ singlet excited states of the spin photoswitch $\left[\mathrm{Fe}(\mathrm{ptz})_{6}\right]\left(\mathrm{BF}_{4}\right)_{2}$ by means of time-dependent density-functional response theory. ${ }^{7-10}$

\section{THEORETICAL BACKGROUND}

Kohn-Sham density-functional theory ${ }^{11-13}$ (KS-DFT) yields the ground-state density $\rho^{\mathrm{KS}}(\mathbf{r})=\sum_{j}^{N=\mathrm{occ}}\left|\psi_{j}^{\mathrm{KS}}(\mathbf{r})\right|^{2}$ and other ground-state properties of an $N$-electron system based on the solution of the Kohn-Sham equations which yield the 


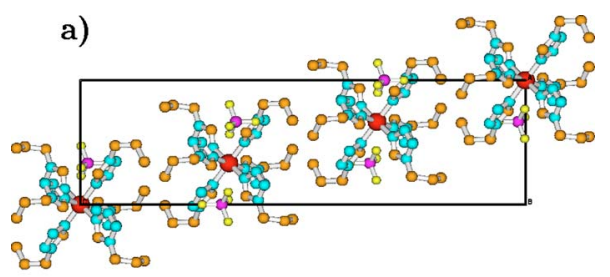

b)

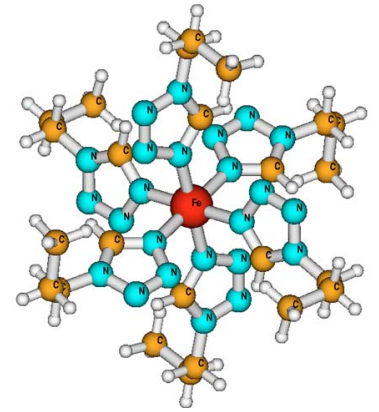

FIG. 1. (Color online) Crystallographic structure of $\left[\mathrm{Fe}(\mathrm{ptz})_{6}\right]\left(\mathrm{BF}_{4}\right)_{2}$ (ptz=1-propyltetrazole) (Ref. 4): (a) Projection along the hexagonal $b$ axis of a complete cell showing some of the Fe and counterion molecules with centers at zero height. (b) Structure of a single $\left[\mathrm{Fe}(\mathrm{ptz})_{6}\right]^{2+}$ cation.

time-independent orbitals $\psi_{j}^{\mathrm{KS}}(\mathbf{r})$. By contrast, timedependent density-functional response theory ${ }^{8-10}$ (TDDFRT) provides a rigorous framework for the computation of excited-state properties and can be applied to finite systems such as single molecules. In particular, TD-DFRT can predict fairly accurate $^{14}$ excitation energies, oscillator strengths, and dipole moments associated with transitions from ground to excited states upon interaction of the system's electronic density with a resonant time-dependent electric perturbation. An starting point of TD-DFRT are the timedependent Kohn-Sham equations which are expressed in terms of an effective potential for a noninteracting system of $N$ electrons, $v_{\text {eff }}(\mathbf{r}, t)$, that explicitly includes a timedependent perturbation $v(\mathbf{r}, t)$ and whose orbitals $\psi_{j}(\mathbf{r}, t)$ yield the dynamic electron density $\rho(\mathbf{r}, t)$ of the truly interacting system, ${ }^{7,15}$

$$
\left[-\frac{1}{2} \nabla^{2}+v_{\mathrm{eff}}[\rho](\mathbf{r}, t)\right] \psi_{j}(\mathbf{r}, t)=i \frac{\partial}{\partial t} \psi_{j}(\mathbf{r}, t),
$$

where $\rho(\mathbf{r}, t)=\sum_{j}^{N}\left|\psi_{j}(\mathbf{r}, t)\right|^{2}$ and the effective potential is given by

$$
v_{\text {eff }}[\rho](\mathbf{r}, t)=v(\mathbf{r}, t)+\int \frac{\rho(\mathbf{r}, t)}{\left|\mathbf{r}-\mathbf{r}^{\prime}\right|} \mathrm{d} \mathbf{r}^{\prime}+v_{\mathrm{xc}}[\rho](\mathbf{r}, t) .
$$

In (2) the exchange-correlation potential accounts for the nonclassical interactions and corresponds to the functional derivative of the exchange-correlation action which, within the adiabatic approximation, is approximated as the exchange-correlation energy of time-independent KohnSham theory ${ }^{11-13}$ evaluated at a particular $t$ :

$$
v_{\mathrm{xc}}[\rho](\mathbf{r}, t)=\frac{\delta A_{\mathrm{xc}}[\rho(\mathbf{r}, t)]}{\delta \rho(\mathbf{r}, t)} \approx \frac{\delta E_{\mathrm{xc}}\left[\rho^{t}(\mathbf{r})\right]}{\delta \rho^{t}(\mathbf{r})}=v_{\mathrm{xc}}\left[\rho^{v}\right](\mathbf{r})
$$

For the computation of polarizabilities, excitation energies, and oscillator strengths only the linear response of the den-

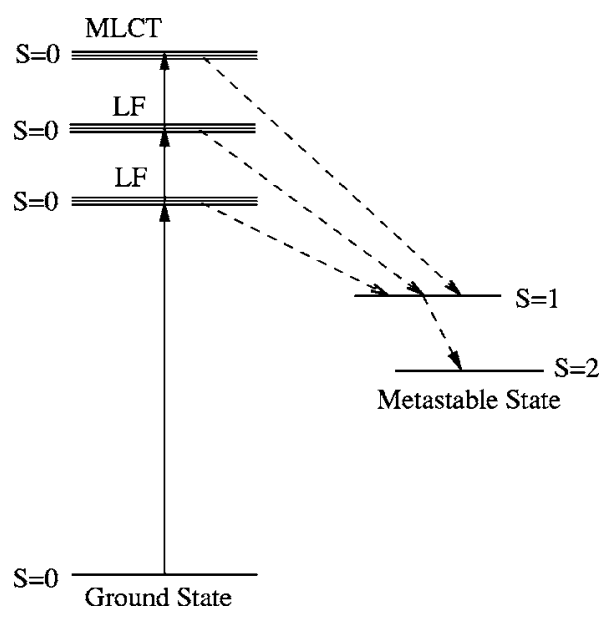

FIG. 2. Basic mechanism of light-induced excited-state spin trapping in $\left[\mathrm{Fe}(\mathrm{ptz})_{6}\right]\left(\mathrm{BF}_{4}\right)_{2}$. Interaction of the $S=0$ multielectronic ground state with light promotes singlet $\rightarrow$ singlet vertical excitations to manifolds of either ligand field (LF) or metal to ligand charge-transfer (MLCT) states which are represented by arrows. The dashed lines represent radiationless spinforbidden intersystem crossings to an intermediate triplet $(S=1)$ and to a metastable quintet $(S=2)$ state.

sity is needed which allows for a perturbative solution to the time-dependent Kohn-Sham equations. The average frequency-dependent dynamic polarizability $\bar{\alpha}(w)$ describes the response of the system's dipole moment to a weak timedependent electric field (perturbation) and can be evaluated from the linear response of the time-dependent electron density. This in turn allows the computation of excited-state energies in the frequency domain $w_{i}$ and corresponding oscillator strengths $f_{i}$, which are shown as poles and residues, respectively, in the sum-over-states expression for the dynamic polarizability: ${ }^{8-10}$

$$
\bar{\alpha}(-w ; w)=\sum_{i} \frac{f_{i}}{w_{i}^{2}-w^{2}}=\frac{2}{3} \sum_{i} \frac{w_{i} \mu_{i}^{2}}{w_{i}^{2}-w^{2}}
$$

In (4) only the dipole-allowed transitions contribute to the summation, ${ }^{16} w_{i} \equiv E_{i}-E_{o}$ are the vertical excitation frequencies, $-w$ corresponds to the sum of these frequencies, and $\mu_{i}^{2}$ are the squared magnitudes of the transition dipole moments. ${ }^{8-10}$ Herein, we report on the ground- and excitedstate properties of the cation of $\left[\mathrm{Fe}(\mathrm{ptz})_{6}\right]\left(\mathrm{BF}_{4}\right)_{2}$ based on time-independent and time-dependent density-functional calculations, respectively.

The Kohn-Sham orbitals which yield the ground-state electron density and have energies $\epsilon_{j}$ are expanded in terms of atom-centered basis functions $\chi_{\mu}(\mathbf{r})$, which mimic atomic orbitals $\psi_{j}^{\mathrm{KS}}(\mathbf{r})=\Sigma_{\mu} c_{\mu j} \chi_{\mu}(\mathbf{r})$. Analysis of the composition of individual molecular orbitals allows the computation of partial density of states (PDOS) contributions from individual atom-centered basis to the total density of states (TDOS). In particular, the partial contribution to the DOS from a basis function $\chi_{\mu}$ can be expressed as $\operatorname{PDOS}_{\mu}(E)$ $=\Sigma_{j} \Sigma_{\nu} c_{\mu j} c_{\nu j}\left\langle\chi_{\nu} \mid \chi_{\mu}\right\rangle \delta\left(E-\epsilon_{j}\right)$ where one substitutes the delta function by broadened Gaussians. ${ }^{17}$ 


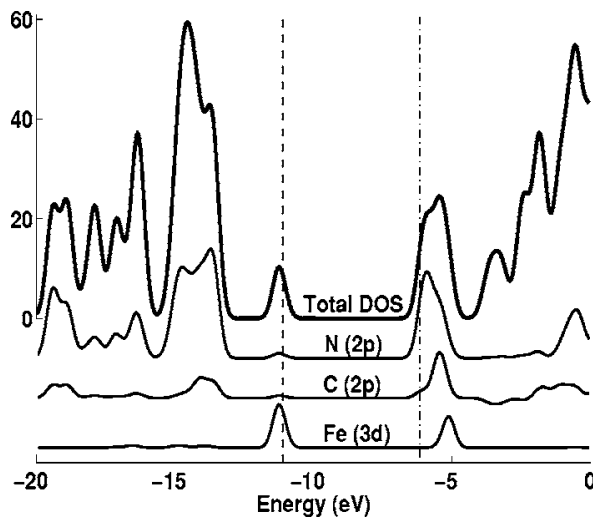

FIG. 3. Total and partial density of states (DOS) around the HOMO-LUMO gap for the multielectronic singlet ground state ${ }^{1} A_{g}$ of $\left[\mathrm{Fe}(\mathrm{ptz})_{6}\right]^{2+}$. The partial DOSs corresponding to contributions of valence shells from the iron ion or tetrazole $(\mathrm{Tz})$ fragments are also shown: $\mathrm{Fe}(3 d), \mathrm{Tz} \mathrm{N}(2 p)$, and $\mathrm{Tz}$ $\mathrm{C}(2 p)$. The dashed vertical lines indicate energies of the HOMO:MO 192 (left) and LUMO: MO 193 (right), respectively. Ground-state DFT calculations used the B3LYP functional (Refs. 18-20) and the all-electron basis $6-311 \mathrm{G}(d, p)$ (Ref. 29) as implemented in GAUSSIAN 98 (Ref. 23).

\section{RESULTS AND DISCUSSION}

The ground-state electronic structure of $\left[\mathrm{Fe}(\mathrm{ptz})_{6}\right]^{2+}$ can be conveniently interpreted in terms of DOS plots as shown in Fig. 3 where the left and right dashed lines correspond to the highest occupied molecular orbital (HOMO) and lowest unoccupied molecular orbital (LUMO) energies, respectively. The partial DOS reveal that the HOMOs are fairly localized on the metal ion with only minor, but nonzero, contributions from nitrogens and carbons of the tetrazole $(\mathrm{Tz})$ rings. By contrast, the lowest unoccupied orbitals (MO 193-204) are highly delocalized throughout the tetrazole rings with almost negligible contributions from Fe-centered orbitals. The localized or delocalized behavior of some representative orbitals is shown in Figs. 4 and 5. In particular, the localized HOMO (MO 192) and delocalized LUMO (MO a)

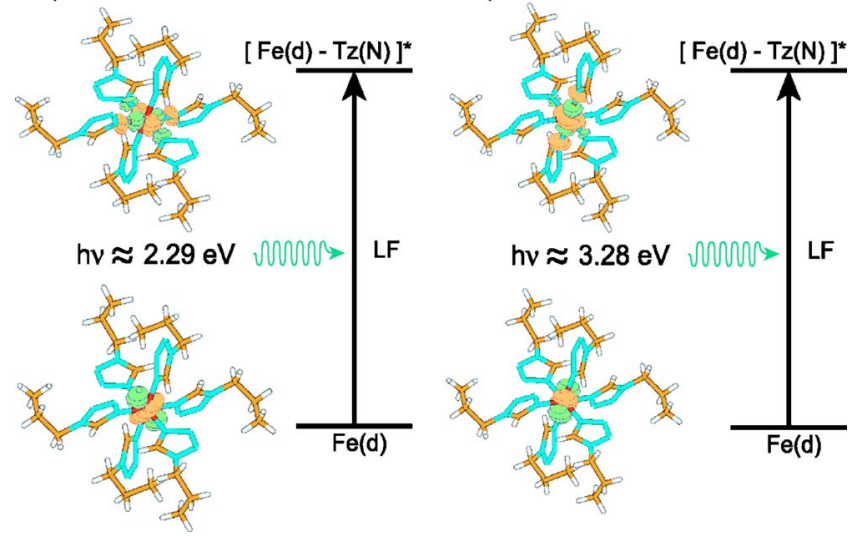

FIG. 4. (Color online) (a) The dominant contribution to the lowest-energy transition shown in Table I: excited state 1 of ligand field (LF) character. An electron excitation occurs from iron-centered MO 192 (HOMO) to unoccupied MO 206 which is mainly an $\mathrm{Fe}(3 d)$ orbital antibonding with $\mathrm{N}(2 p)$ orbitals of four neighboring tetrazole nitrogens. (b) One of the dominant contributions to the lower-energy transitions shown in Table I: excited states 1,3 , and 5 of ligand field (LF) character. An electron excitation from ironcentered MO 191 to unoccupied MO 205 which is mainly an $\mathrm{Fe}(3 d)$ orbital antibonding with $\mathrm{N}(2 p)$ orbitals of two neighboring tetrazole nitrogens. a)

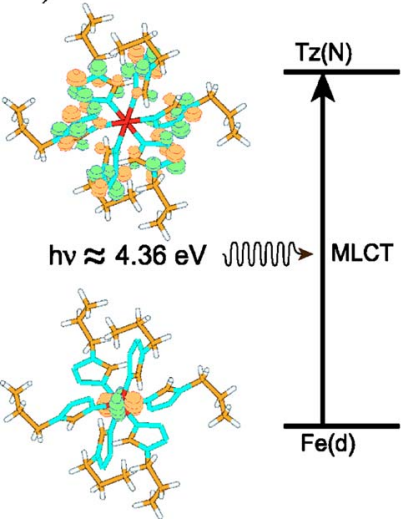

b)

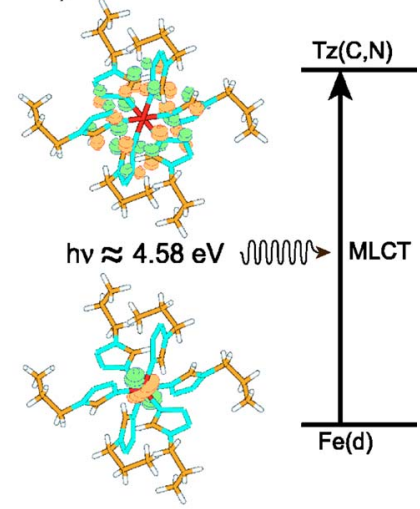

FIG. 5. (Color online) (a) The dominant contribution to a formally allowed MLCT transition (excited state 13, $f=0.0078$ ). An electron excitation occurs from iron-centered MO 190 to unoccupied MO 193 (LUMO) which is delocalized throughout the tetrazole rings. (b) The dominant contributions to the MLCT transition with largest oscillator strength (excited state 23, $f=0.1435$ ). An excitation occurs from iron-centered MO 192 (HOMO) to unoccupied MO 199 which is delocalized throughout $\mathrm{Tz}(\mathrm{C}, \mathrm{N})$ orbitals.

193) are shown in Figs. 4(a) and 5(a), respectively. Slightly above the LUMO energy, however, some orbitals (MO 205 and 206) have main $\mathrm{Fe}(3 d)$ character with non-negligible contributions from tetrazole rings which are antibonding to the metal [Figs. 4(a) and 4(b)]. We notice that this particular ordering of the unoccupied frontier orbitals (LUMOs) is somewhat dependent on the chosen exchange-correlation functional (B3LYP) (Refs. 18-20) which is here used to describe ground- and excited-state electronic structures. The use of an alternative functional (BPW91) (Refs. 21 and 22) yielded a somewhat differing ordering whereby the lowestenergy LUMOs are metal based and those slightly higher in energy are tetrazole based. However, as described below, the basic qualitative description of the excited-state properties via TD-DFRT is the same for both types of representative functionals. To confirm the results, ground-state calculations were carried out for $C_{i}$ and $S_{6}$ symmetries with two representative electronic structure packages (GAUSSIAN 98: ${ }^{23}$ B3LYP/6-311G ${ }^{*}$ and JAGUAR 4.2: ${ }^{24}$ B3LYP/LACVP $\left.{ }^{* *}\right)$. The qualitative results obtained from both packages were identical. Excited-state calculations were done within the $C_{i}$ group with GAUSSIAN $98 .^{23}$

The ground-state properties described above provide a useful reference for understanding the excited states of the complex within the framework of TD-DFRT. ${ }^{8-10}$ Here, excited states are expanded in terms of several single-electron excitations from particular occupied to particular unoccupied Kohn-Sham orbitals as shown in Table I. All the low-energy transitions with finite oscillator strength involve excitations from Kohn-Sham orbitals of even symmetry to those of odd symmetry $(g \rightarrow u)$. This is true for orbitals obtained from ground-state calculations within the $C_{i}$ and $S_{6}$ symmetry groups and whose respective labels are given in Table II. In particular, the lowest-energy excited state with finite oscillator strength (excited state 7) corresponds to a transition from the HOMO (MO 192) with symmetry label $A_{g}$ to the LUMO (MO 193) with label $A_{u}$ (Tables I and II). The basic qualitative description of the excitations with finite oscillator 
TABLE I. Properties of selected vertical singlet excited states of $\left[\mathrm{Fe}(\mathrm{ptz})_{6}\right]^{2+}$ obtained from TD-DFRT. Calculations used the B3LYP functional and the all-electron basis $6-31 \mathrm{G}^{*}$ as implemented in GAUSSIAN 98 (Ref. 23).

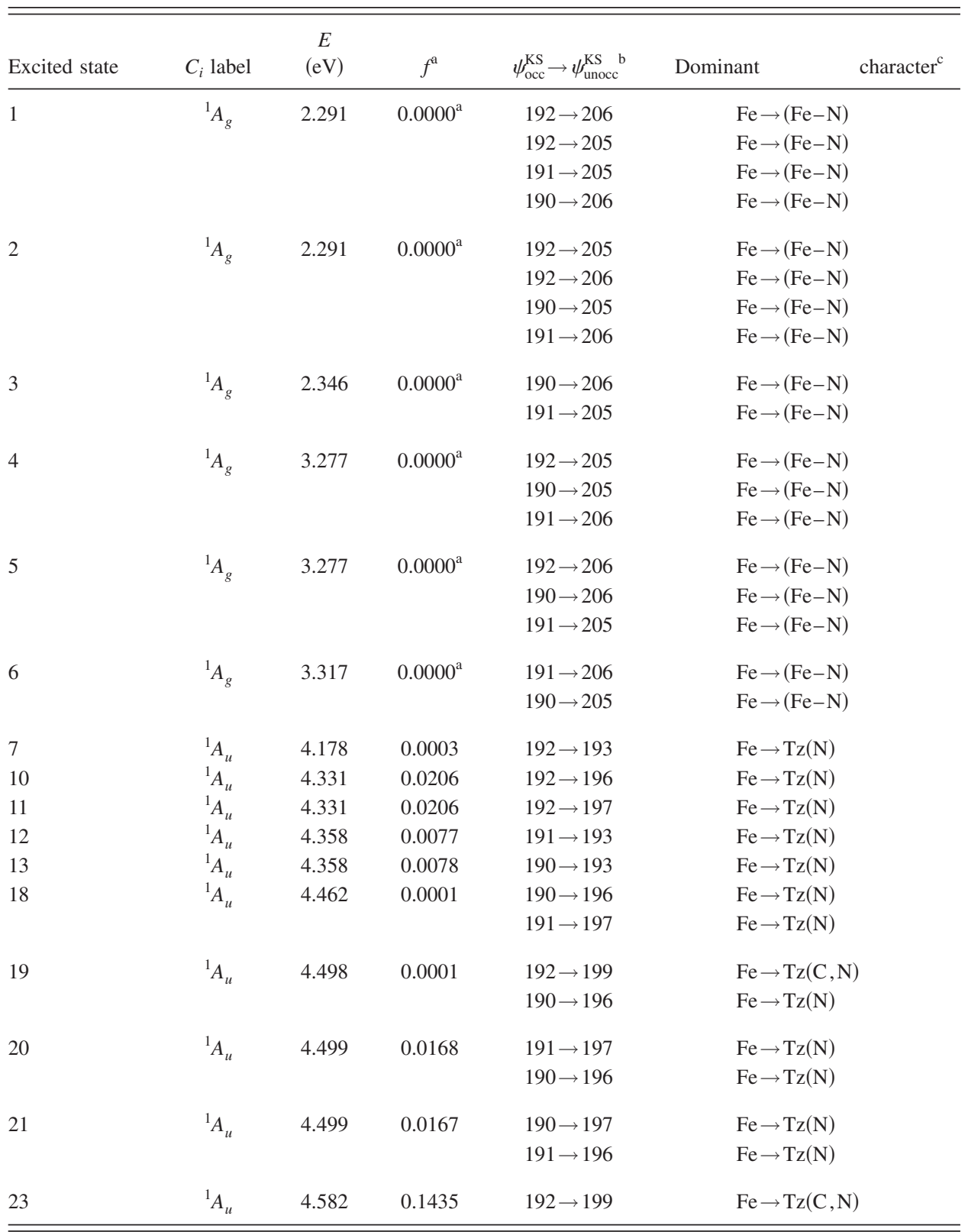

$\overline{{ }^{a} \text { Values obtained for } C_{i} \text { symmetry. A minor change of a single Fe-N bond lowers the symmetry to } C_{1} \text { which }}$ makes the formally zero oscillator strengths to acquire small values $\left(10^{-3}-10^{-4}\right)$.

${ }^{\mathrm{b}}$ Only the dominant single-electron excitations which contribute to a particular excited state are shown in order of importance. Some excited states have several dominant such contributions and others, e.g., excited state 7, have only one.

${ }^{c}(\mathrm{Fe}-\mathrm{N})$ indicates unoccupied antibonding MOs with a dominant $\mathrm{Fe}(3 d)$ contribution which is antisymmetric to a minor contribution from neighboring $\mathrm{N}(p)$ orbitals. $\mathrm{Tz}(\mathrm{N})$ or $\mathrm{Tz}(\mathrm{C})$ indicate unoccupied MOs with dominant tetrazole $\mathrm{N}(p)$ or tetrazole $\mathrm{C}(p)$ contribution, respectively.

strength is the same for B3LYP (Refs. 18-20) and BPW91 (Refs. 21 and 22) (not shown) functionals even though the relative ordering of their unoccupied Kohn-Sham orbitals is somewhat different. Thus, for both functionals, the lowestenergy excited state with formally finite oscillator strength mainly corresponds to a $\mathrm{HOMO}\left(\mathrm{MO} 192, A_{g}\right)$ $\rightarrow$ LUMO(MO 193, $\left.A_{u}\right)$ transition.

Although single-crystal absorption spectra of $\left[\mathrm{Fe}(\mathrm{ptz})_{6}\right]$ $\times\left(\mathrm{BF}_{4}\right)_{2}$ display rather broad and unresolved bands around $2.28 \mathrm{eV}\left(\approx 18400 \mathrm{~cm}^{-1}\right)$ and $3.30 \mathrm{eV}\left(\approx 26650 \mathrm{~cm}^{-1}\right){ }^{1}$ contrary to simpler models, ${ }^{1,2,6}$ the time-dependent calcula- tions revealed that each of these bands is a complex superposition of several singlet $\rightarrow$ singlet excitations which are closely packed on the energy scale. The computed excitation energies are given in Table I and very closely agree with the experimental values. In particular, excited states 1 and 2 where calculated at $2.29 \mathrm{eV}$ with respect to the ground state in remarkable agreement with the measured energy of the first absorption band around $2.28 \mathrm{eV}{ }^{1}$ Similarly, excited states 4 and 5 were calculated at $3.28 \mathrm{eV}$ above the ground state in close agreement with the measured value for the second band around $3.30 \mathrm{eV} .{ }^{1}$ Recent TD-DFRT calculations 
TABLE II. Labels of relevant ground-state Kohn-Sham orbitals for $C_{i}$ and $S_{6}$ symmetry groups.

\begin{tabular}{lccc}
\hline \hline$\psi^{\mathrm{KS}}$ & Type & $C_{i}$ & $S_{6}$ \\
\hline 190 & Occupied & $A_{g}$ & $E g$ \\
191 & Occupied & $A_{g}$ & $E g$ \\
192 & Occupied & $A_{g}$ & $\mathrm{Ag}$ \\
193 & Unoccupied & $A_{u}$ & $\mathrm{Au}$ \\
196 & Unoccupied & $A_{u}$ & $\mathrm{Eu}$ \\
197 & Unoccupied & $A_{u}$ & $\mathrm{Eu}$ \\
199 & Unoccupied & $A_{u}$ & $\mathrm{Au}$ \\
205 & Unoccupied & $A_{g}$ & $\mathrm{Eg}$ \\
206 & Unoccupied & $A_{g}$ & $\mathrm{Eg}$ \\
\hline \hline
\end{tabular}

have also shown close agreement with experiment for a related iron complex. ${ }^{14}$ Computed excited states 1-3 and 4-6 (Table I) are, therefore, identified with the 2.28 and $3.30 \mathrm{eV}$ absorption bands of the experimental spectrum, respectively. Furthermore, the TD-DFRT calculations show that these bands do not correspond to excitations from the ground to a single excited state but that each of these is a superposition of nonresolved intensities corresponding to some three excited states which are close on the energy scale. These six lower-energy transitions 1-3 and 4-6, have main ligand field $(\mathrm{LF}), \mathrm{Fe}(3 d) \rightarrow \mathrm{Fe}(3 d)$, character as illustrated by Figs. 4(a) and 4(b) whereby metallic electron density is transferred to higher-energy states dominated by antisymmetric (antibonding) combinations of $\mathrm{Fe}(3 d)$ orbitals and small contributions from tetrazole nitrogen orbitals. The substantial ligand field (LF) character predicted for these transitions is also consistent with their lower intensities and lower energies in the experimental spectrum relative to those measured around 4.18-4.58 eV which, as described below, are clearly of metal to ligand character and, therefore, expected to be more intense and higher in energy. Table I shows the computed energies and other properties of the most relevant singlet $\rightarrow$ singlet excitations. The crystallographic structure of a single molecular complex very closely corresponds to $S_{6}$ symmetry with the largest Abelian subgroup being $C_{i}$ in which the iron is the center of inversion. The $C_{i}$ molecular symmetry used in the KS-DFT and TD-DFRT calculations gave rise to a ${ }^{1} A_{g}$ multielectronic ground state which formally does not produce finite transition dipole moments with the lower-energy excited states of the same symmetry. For centrosymmetric molecules with inversion symmetry, transitions of equal parity $(g \rightarrow g)$ are formally forbidden whereas those of different parity $(g \rightarrow u)$ are allowed. Accordingly, the six lowest-energy transitions (i.e., excited states 1-6) yielded a formally zero oscillator strength $(f=0)$ which, however, became finite upon lowering the molecular symmetry to $C_{1}$. In fact, shortening one of the $\mathrm{Fe}-\mathrm{N}$ bonds by $\approx 0.1 \AA$ introduced a slight distortion which produced small but finite oscillator strengths $\left(f \approx 10^{-3}-10^{-4}\right)$ for excitation energies around $2.3-3.3 \mathrm{eV}$ corresponding to excited states $1-6$. We are further studying the origin of the finite LF intensities observed in the experimental spectrum. However, comparison of our TD-DFRT calculations performed with $C_{i}$ and $C_{1}$ symmetry strongly suggests that the lower-energy LF bands originate from meaningful distortions from the nomi- nal $C_{i}$ symmetry. It is known that formally forbidden $g \rightarrow g$ transitions can become allowed if the center of symmetry is eliminated during asymmetric vibrations. Thus, the distortions from the nominal symmetry most likely originate in the coupling of electronic and nuclear degrees of freedom (vibronic coupling) which has been shown to yield sizable absorption intensities for otherwise parity-forbidden transitions within the context of TD-DFRT. ${ }^{25}$ Other studies on related LIESST crystals have also shown that coupling of the electronic state and the phonon system plays a dominant role on their phase transitions. ${ }^{26,27}$ In addition, crystal packing forces may lower the molecular symmetry relative to the gas-phase calculations reported here further promoting absorption intensity.

For $C_{i}$ symmetry all the lower-energy transitions with formally finite oscillator strength (excited states $7,10,11, \ldots$, Table I) clearly correspond to singlet $\rightarrow$ singlet metal to ligand charge transfers (MLCT). Some representative examples are shown in Figs. 5(a) and 5(b). Most of these nearly degenerate excited states had a dominant $\mathrm{Fe}(3 d) \rightarrow \mathrm{Tz}(\mathrm{N})$ character whereby metal-centered electron density was mainly transferred to the nitrogens of the tetrazole rings. The most intense MLCT transition (excited state 23, $f=0.1435$ ), however, corresponds to transfer of metallic electron density to an excited state of dominant $\mathrm{Tz}(\mathrm{C}, \mathrm{N})$ character. By lowering the molecular symmetry to $C_{1}$ these metal to ligand excitations also produced a somewhat greater oscillator strength. The experimental spectrum recorded at $8 \mathrm{~K}$ shows that the two lower-energy LF bands have extinction coefficients of $\gtrsim 201 / \mathrm{mol} \mathrm{cm}$ and $\lesssim 301 / \mathrm{mol} \mathrm{cm}$, respectively, whereas that of the MLCT band is presumably much larger since it falls outside the range of the published data. ${ }^{1,6}$ This is qualitatively consistent with the small oscillator strength we estimate (Table I) for the LF bands within $C_{1}$ symmetry $(f$ $=10^{-3}-10^{-4}$ ) and with the substantially higher oscillator computed for the most intense MLCT excitation (excited state $23, f=0.1435)$.

The excited-state properties determined from the present TD-DFRT calculations provide insight on the initial mechanisms of light-induced excited-state spin trapping (LIESST). The lower-energy absorption bands observed experimentally around 2.28 and $3.30 \mathrm{eV}$ originate in ligand field (LF) transitions which transfer electron density from localized $\mathrm{Fe}(3 d)$ orbitals to antibonding states of main $\mathrm{Fe}(3 d)$ character with additional $\quad \mathrm{Tz}(\mathrm{N}) \quad$ contributions: $\quad \mathrm{Fe}(3 d) \rightarrow[\mathrm{Fe}(3 d)$ $-\mathrm{Tz}: \mathrm{N}(2 s, 2 p)]^{*}$. As shown in Figs. 4(a) and 4(b), the minor $\mathrm{Tz}(\mathrm{N})$ contributions to these antibonding states are localized on the nitrogens which are chemically bound to the iron. The antibonding nature of the metal-ligand interactions in these excited states in turn weakens the $\mathrm{Fe}-\mathrm{N}$ bonds and promotes their elongation. Although the crystallographic structures of the ground singlet and metastable quintet states of $\left[\mathrm{Fe}(\mathrm{ptz})_{6}\right]\left(\mathrm{BF}_{4}\right)_{2}$ show dramatic structural rearrangements and $\mathrm{Fe}-\mathrm{N}$ bond lengths which are about $0.2 \AA$ longer for the latter state, no clear microscopic understanding exists about the origin of such elongations. The present results show that electromagnetic absorption close to the green region $(2.28-3.30 \mathrm{eV})$ is inherently associated with the formation of vertical antibonding excited states which promote elonga- 
tion of the $\mathrm{Fe}-\mathrm{N}$ bonds. This follows since to reach equilibrium in the excited state the nuclear coordinates must, in general, relax. Therefore, the structural rearrangements crystallographically detected for the metastable quintet likely begin to appear before this state is actually formed, namely, at the beginning stages of the LIESST process when the antibonding singlets are formed and, thereafter, at the initial (singlet-triplet) intersystem crossing. This is consistent with recent spectroscopic studies suggesting that the ligand field $\mathrm{Fe}(3 d) \rightarrow \mathrm{Fe}(3 d)$ transitions more efficiently drive the cooperative change in spin state than other excitation bands ${ }^{27}$ and also with our own geometry optimizations for the intermediate triplet state which show longer $\mathrm{Fe}-\mathrm{N}$ bonds relative to the ground-state singlet. ${ }^{28}$

\section{CONCLUSION}

We have obtained the first detailed microscopic description of the ground- and excited-state electronic structures of the cation of the LIESST-exhibiting $\left[\mathrm{Fe}(\mathrm{ptz})_{6}\right]\left(\mathrm{BF}_{4}\right)_{2}$ spin photoswitch. The complex is a prototype of a wider class of spin-forbidden crossover compounds and the qualitative insight gained here should apply to other such systems. Although there have been many experimental studies on the ground and metastable states of the complex, only limited theoretical information existed about the properties of its excited states. The present time-dependent calculations on the complete cationic molecular structure have provided a detailed microscopic description of the vertical singlet $\rightarrow$ singlet excitations which are observed in the experimental spectrum. The energies of the LF and higher-energy MLCT excitations have been calculated with remarkable accuracy relative to the experimental spectra. The essentially centrosymmetric crystalline structure of the cationic complex formally produces no or very weak ligand field $\mathrm{Fe}(3 d)$ $\rightarrow \mathrm{Fe}(3 d)$ absorption intensities of strict electronic origin. Therefore, the formally parity-forbidden lower-energy LF bands mainly arise from vibronic transitions due to coupling of the electronic and phonon systems. Furthermore, such LF bands are not simple singlet $\rightarrow$ singlet excitations but arise from ground-state transitions to two manifolds of nearly degenerate excited states (1-3 and 4-6). Finally, the antibonding nature of the singlet LF excited states naturally promotes $\mathrm{Fe}-\mathrm{N}$ bond elongations which strongly suggest that the dilation of the ligand cage surrounding the iron at the metastable quintet likely begins to appear at the onset of the LS $\rightarrow \mathrm{HS}$ transition process, namely, at the optically or thermally induced occupation of the singlet antibonding states.

\section{ACKNOWLEDGMENTS}

Research funded by NSF Career Award CHE-0349189 (JHR) and Purdue University Research Foundation. Computer time provided by Purdue's ITaP and the National Center for Supercomputer Applications (NCSA) is appreciated. The author thanks Fredy Aquino, Teepanis Chachiyo, and Taisuke Minagawa for assistance preparing the manuscript.

${ }^{1}$ S. Decurtins, P. Gutlich, K. M. Hasselbach, A. Hauser, and H. Spiering, Inorg. Chem. 24, 2174 (1985).

${ }^{2}$ P. Gutlich, Y. Garcia, and T. Woike, Coord. Chem. Rev. 219, 839 (2001).

${ }^{3}$ L. Wiehl, H. Spiering, P. Gütlich, and K. Knorr, J. Appl. Crystallogr. 23, 151 (1990).

${ }^{4}$ L. Wiehl, Acta Crystallogr. 49, 289 (1993).

${ }^{5}$ O. Kahn, Molecular Magnetism (VCH, New York, 1993).

${ }^{6}$ A. Hauser, A. Vef, and P. Adler, J. Chem. Phys. 95, 8710 (1991)

${ }^{7}$ E. K. U. Gross and W. Kohn, Adv. Quantum Chem. 21, 255 (1990).

${ }^{8}$ M. E. Casida, in Recent Advances in Density Functional Methods, edited by D. P. Chong (World Scientific, Singapore, 1995).

${ }^{9}$ M. E. Casida, in Recent Developments and Applications of Modern Density Functional Theory, Theoretical and Computational Chemistry, edited by J. M. Seminario (Elsevier, Amsterdam, 1996).

${ }^{10}$ C. Jamorski, M. E. Casida, and D. R. Salahub, J. Chem. Phys. 104, 5134 (1996).

${ }^{11}$ W. Kohn and L. J. Sham, Phys. Rev. 140, A1133 (1965).

${ }^{12}$ R. G. Parr and W. Yang, Density-Functional Theory of Atoms and Molecules (Clarendon, Oxford, 1989).

${ }^{13}$ W. Kohn, A. D. Becke, and R. G. Parr, J. Phys. Chem. 100, 12974 (1996).

${ }^{14}$ J. E. Monat, J. H. Rodriguez, and J. K. McCusker, J. Phys. Chem. A 106, 7399 (2002).

${ }^{15}$ E. Runge and E. K. U. Gross, Phys. Rev. Lett. 52, 997 (1984).

${ }^{16}$ S. J. A. van Gisbergen, J. G. Snijders, and E. J. Baerends, Comput. Phys. Commun. 118, 119 (1999).

${ }^{17}$ F. Aquino and J. H. Rodriguez, Proceedings of the Molecular Orbital Density of States Program, West Lafayette, IN, 2005 (unpublished).

${ }^{18}$ A. D. Becke, J. Chem. Phys. 98, 1372 (1993).

${ }^{19}$ A. D. Becke, J. Chem. Phys. 98, 5648 (1993).

${ }^{20}$ C. Lee, W. Yang, and R. G. Parr, Phys. Rev. B 37, 785 (1988).

${ }^{21}$ A. D. Becke, Phys. Rev. A 38, 3098 (1988).

${ }^{22}$ J. P. Perdew and Y. Wang, Phys. Rev. B 45, 13244 (1992).

${ }^{23}$ M. J. Frisch, G. W. Trucks, H. B. Schlegel et al., GAUSSIAN 98, Revision A.4, Gaussian, Inc., Pittsburgh PA, 1998.

${ }^{24}$ JAGUAR 4.2, Schrodinger, Inc., Portland, Oregon, 2000.

${ }^{25}$ G. F. Bertsch and A. Schnell, J. Chem. Phys. 115, 4051 (2001)

${ }^{26}$ M. Sorai and S. Seki, J. Phys. Chem. Solids 35, 555 (1974).

${ }^{27}$ Y. Ogawa, S. Koshihara, K. Koshino, T. Ogawa, C. Urano, and H. Takagi, Phys. Rev. Lett. 84, 3181 (2000).

${ }^{28}$ T. Chachiyo and J. H. Rodriguez (unpublished).

${ }^{29}$ W. J. Hehre, R. Ditchfield, and J. A. Pople, J. Chem. Phys. 56, 2257 (1972). 